\title{
International Symposium on Macro- and Supramolecular Architectures and Materials (MAM-17) June 6-10, 2017
}

IUPAC sponsored the International Symposium on Macro- and Supramolecular Architectures and Materials (MAM-17): Multifunctional Materials and Structures is organized by the Lomonosov Moscow State University (Moscow, Russia) and the Southern Federal University (Rostov, Russia). It will be held in Sochi (June 6-10, 2017).

$\begin{array}{ll}\text { Honorary Chairman } & \text { V. Sadovnichiy, Rector of the Lomonosov Moscow State University (Russia) } \\ \text { Chairs } & \text { Prof. E. Karakhanov, Lomonosov Moscow State University (Russia) } \\ & \text { Prof. K. Geckeler, Gwangju Institute of Science and Technology (South Korea) }\end{array}$

International Advisory Board

E. Alsharaeh (Saudi Arabia)
M. Attia (Egypt)
O. Awitor (France)
C. Bai (China)
G. Cuniberti (Germany)
G. Dzhardimalieva (Russia)
H. Fuchs (Germany)
E. Gillies (Canada)
B. B. Gupta (India)
I. Kahwa (Jamaica)
S. Kudaibergenov (Kazakhstan)
J. M. Irache (Spain)
R. Laine (USA)
Yu. Lvov (USA)
M. Maaza (S. Africa)
A. Maximov (Russia)
V. Minkin (Russia)
M. Mohan (USA)
K. Muellen (Germany)
W. Nau (Germany)
H. Nishide (Japan)
T. Premkumar (S. Korea)
H. Pasch (S. Africa)
S. Penczek (Poland)
S. Ramakrishna (Singapore)
V. Rajendran (India)
E. Rosenberg (USA)
B. Rivas (Chile)

\section{Local Committee}

K. Shin (S. Korea)

J. Soares (Canada)

B. Spivakov (Russia)

N. Tarasova (Russia)

L. Tavadyan (Armenia)

R. Tshikhudo (S. Africa)

V. Vinokurov (Russia)

M. Yallapu (USA)

K. Yamamoto (Japan)

N.L. Yang (USA)

Y. Wu (Chinagapore)

A. Yaroslavtsev (Russia)
Chair: A. Maximov
S. Egazaryants
M. Terenina
V. Shtepa
E. Lokteva
Y. Kardasheva
A. Akopyan
A. Volkov
I. Vatsuro
N. Petruhina
E. Sulman
A.Anisimov
Program Committee
$\begin{array}{ll}\text { Chair: } & \text { V. Lunin } \\ & \text { V. Kovalev }\end{array}$
V. Kukushkin
V. Matveenko
E. Milaeva
A. Maximov
M. Melnikov
O. Sinyashin

The symposium will address the following topics:

- Catalysis and applications

- Membrane technologies

- Energy conversion and storage

- Materials for next generation manufacturing processes

- Biomedicine and bio-engineering
- Macromolecular design

- Engineered materials with high performance

- Nanomaterials and multifunctions

- Chemo- and biosensors

- Environmental applications

- General topics

Individuals, responsible for supplying relevant information

Eduard Karakhanov, Lomonosov Moscow State University, Faculty of Chemistry, Russia, 119991, Moscow 1, GSP-1, 1-3 Leninskiye Gory. E-mail: kar@petrol.chem.msu.ru; fax: +7 (495) 932 8846; tel: +7 (495) 9395377 Anton Maximov, Lomonosov Moscow State University, Faculty of Chemistry, Russia, 119991, Moscow 1, GSP-1, 1-3 Leninskiye Gory. E-mail: max@ips.ac.ru; fax: +7 (495) 9328846.

\section{www.mam-17.org}

